\title{
Argos, fouilles de l'Aspis - 2016
}

Anna Philippa-Touchais, Gilles Touchais et Maria Choleva

\section{OpenEdition \\ Journals}

Édition électronique

URL : http://journals.openedition.org/baefe/1331

DOI : 10.4000/baefe.1331

ISSN : 2732-687X

Éditeur

ResEFE

\section{Référence électronique}

Anna Philippa-Touchais, Gilles Touchais, Maria Choleva, « Argos, fouilles de l'Aspis - 2016 » [notice archéologique], Bulletin archéologique des Écoles françaises à l'étranger [En ligne], Grèce, mis en ligne le 26 novembre 2020, consulté le 22 mars 2021. URL : http://journals.openedition.org/baefe/1331 ; DOI : https://doi.org/10.4000/baefe.1331

Ce document a été généré automatiquement le 22 mars 2021

\section{c) (1) (9)}

Le Bulletin archéologique des Écoles françaises à l'étranger est mise à disposition selon les termes de la Licence Creative Commons Attribution - Pas d'Utilisation Commerciale - Pas de Modification 4.0 International. 


\title{
Argos, fouilles de l'Aspis - 2016
}

\author{
Anna Philippa-Touchais, Gilles Touchais et Maria Choleva
}

\section{NOTE DE L'AUTEUR}

\section{Établissements porteurs de l'opération : EFA}

\section{Données scientifiques produites : Argos par l'EFA}

1 La campagne d'étude s'est déroulée au musée d'Argos entre les mois de juillet et de septembre, alors que l'ensemble du matériel, déjà conditionné en vue du déménagement des réserves ${ }^{1}$, était toutefois demeuré accessible. Dans le cadre de la préparation du premier volume de la publication des fouilles de l'Aspis, consacré aux occupations d'époque historique, on a entrepris l'étude de la seule catégorie d'artefacts qui n'avait pas encore été examinée : celle des outils liés aux activités textiles². On a d'autre part poursuivi l'étude de la céramique mésohelladique, notamment du point de vue technologique, avec l'examen des premières céramiques tournées par M. Choleva, dont nous reproduisons ci-dessous le rapport:

2 L'étude technologique entreprise cette année, selon les principes méthodologiques déjà exposés ${ }^{3}$, avait trois objectifs : 1 ) déterminer la phase d'apparition du tour de potier dans les traditions céramiques de l'Aspis ; 2) corréler son usage à des types morphostylistiques spécifiques et identifier les pratiques techniques impliquées ; 3) esquisser l'impact du tour sur les modes de production traditionnels.

3 Pour ce faire, l'étude s'est concentrée sur les phases anciennes de l'occupation du site, en particulier sur le matériel céramique provenant de la maison MI (secteur Nord), qui date de l'HM II ${ }^{4}$. Une étude comparative, qui n'a pas pu être achevée cette année, a été aussi menée sur la céramique de la «maison fantôme» (secteur Sud-Est) ${ }^{5}$, datée de la fin de l'HMI. 


\section{La céramique de la maison MI}

4 L'ensemble de la céramique de cette maison a été étudié, soit 739 tessons conservés par les fouilleurs à l'issue du tríi. Sur la base de l'usage du tour de potier, deux groupes techniques ont été distingués.

\section{Céramique élaborée au tour}

5 L'usage du tour de potier, c'est-à-dire de l'Énergie Cinétique Rotative (ECR), a été constaté sur un pourcentage minime - de l'ordre de $8 \%$ - de la totalité de la céramique étudiée. Dans tous les cas, la technique mise en œuvre est celle de l'élaboration au tour d'une ébauche préalablement faite aux colombins (fig. 1) ${ }^{7}$. Une seule et unique méthode d'emploi du tour a été identifiée dans la séquence opératoire du façonnage : une fois l'ébauchage aux colombins achevé par pressions discontinues, l'ECR est exploitée pendant la phase finale du façonnage, c'est-à-dire pendant la mise en forme de l'ébauche et la régularisation des surfaces (fig. 2). Son emploi facilite la mise en forme soit de l'ébauche entière du pot, soit de la partie supérieure (col, épaule, bord). Les pots élaborés au tour sont produits par des chaînes opératoires comprenant une série de pratiques techniques variées : 1) lissage fréquent des surfaces à l'aide de l'ECR suivi par un polissage sans l'aide de l'ECR ; 2) application rare d'un engobe ; 3) ajout rare de motifs peints sur les surfaces; 3) emploi de pâtes fines et bien épurées, rarement de pâtes semi-grossières ; 4) cuisson fréquente des pots en atmosphère réductrice qui donne aux surfaces et au biscuit une couleur grise uniforme, la cuisson oxydante étant rare. L'usage du tour de potier a été identifié sur un éventail de types morphologiques restreint. Il s'agit surtout de formes ouvertes de petites ou de moyennes dimensions : canthares carénés, jattes carénées à épaule cannelée ou non, bols renflés et, rarement, gobelets à pied. L'usage du tour pour le façonnage de formes fermées de plus grandes dimensions, comme les jarres à col resserré, est rare et toujours limité à l'élaboration des parties supérieures (surtout le col), le corps étant fabriqué manuellement (fig. 3). Quant aux catégories stylistiques, la technique de l'élaboration au tour se rencontre, de façon très majoritaire, au sein de la céramique grise fine polie (minyenne), surtout pour la fabrication des canthares et des bols renflés, plus rarement pour celle des jattes. Elle est rare, au contraire, dans la céramique à peinture mate, englobant surtout les jattes à épaule non cannelée pour lesquelles l'usage du tour semble limité à l'élaboration des parties supérieures ${ }^{8}$. Enfin, elle se rencontre de façon très marginale au sein de la céramique lustrée, concernant seulement quelques jarres dont seul le col (resserré) est mis en forme à l'aide du tour (fig. 4). Il semble qu'il existe une liaison étroite entre les bols/canthares minyens et l'ECR, tandis que l'exploitation de celle-ci pour le façonnage des autres types morpho-stylistiques demeure occasionnelle. C'est le cas, par exemple, des jattes carénées à épaule cannelée, qui sont très bien représentées dans la céramique à Aspis. Cette forme, en minyen gris mais surtout en céramique noire lissée, est produite de façon systématique par des techniques manuelles, en particulier celle des colombins. 
Fig. 1. Tesson minyen 79/351.20.

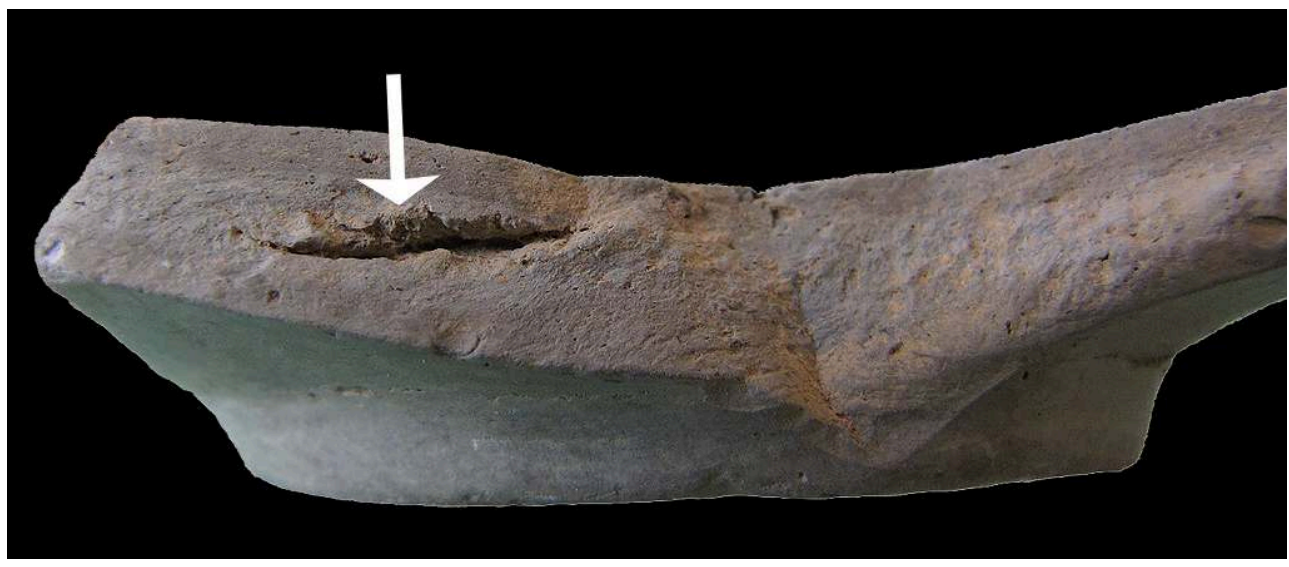

Section, joint de colombins.

EFA.

Fig. 2. Tesson minyen 83/635.15.

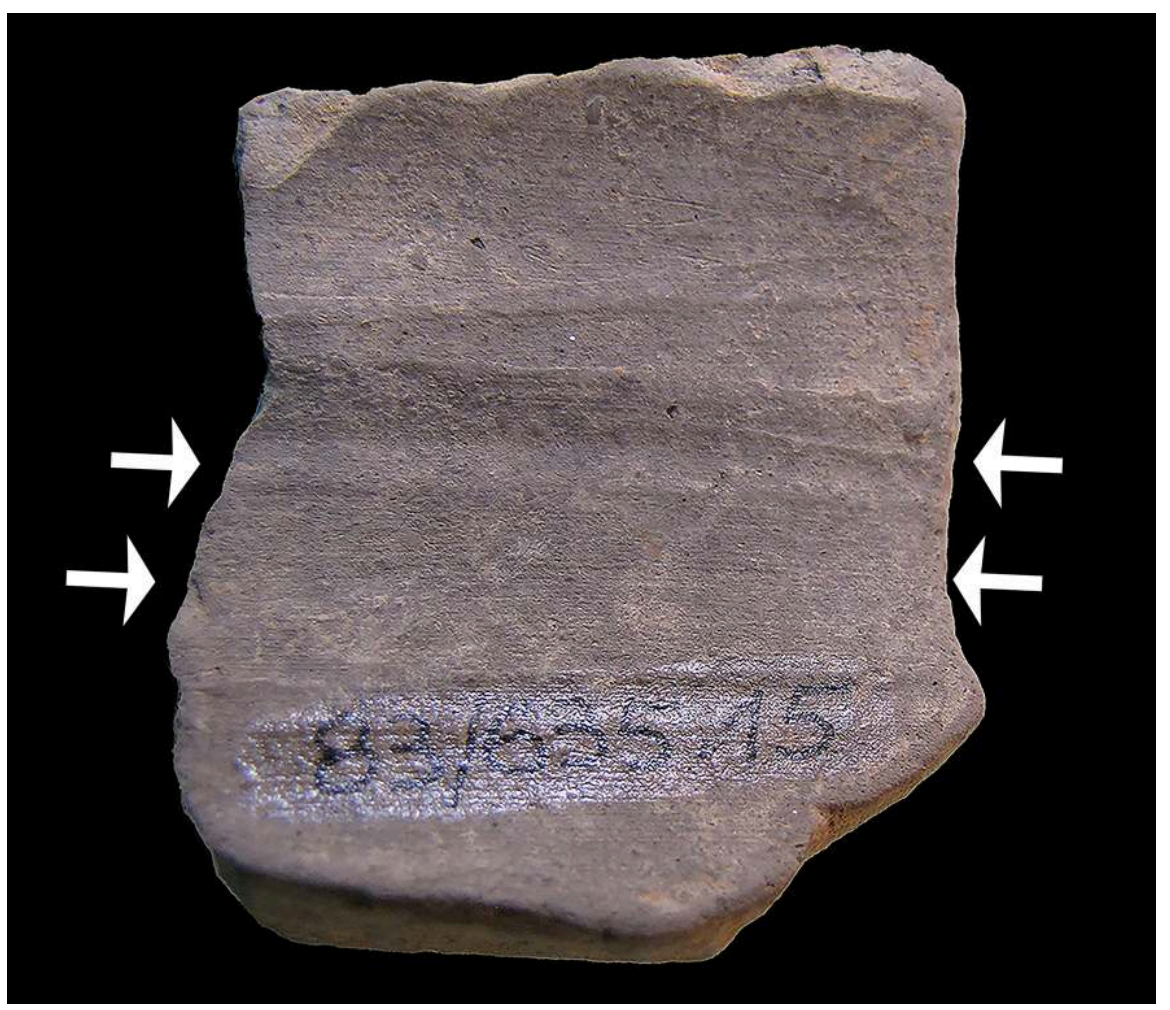

Surface interne, stries horizontales parallèles issues de l'ECR et microrelief irrégulier issu des pressions discontinues.

EFA. 
Fig. 3. Types morphologiques de la céramique élaborée au tour dans la maison MI.

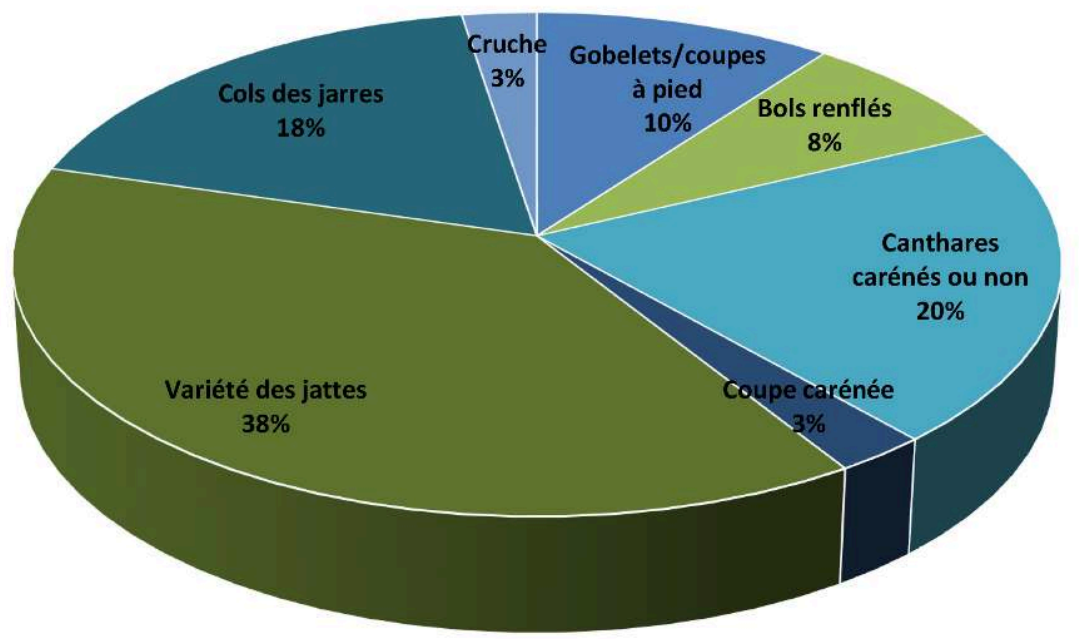

EFA.

Fig. 4. Répartition des pièces portant des traces de l'ECR dans les catégories céramiques de la maison Ml.

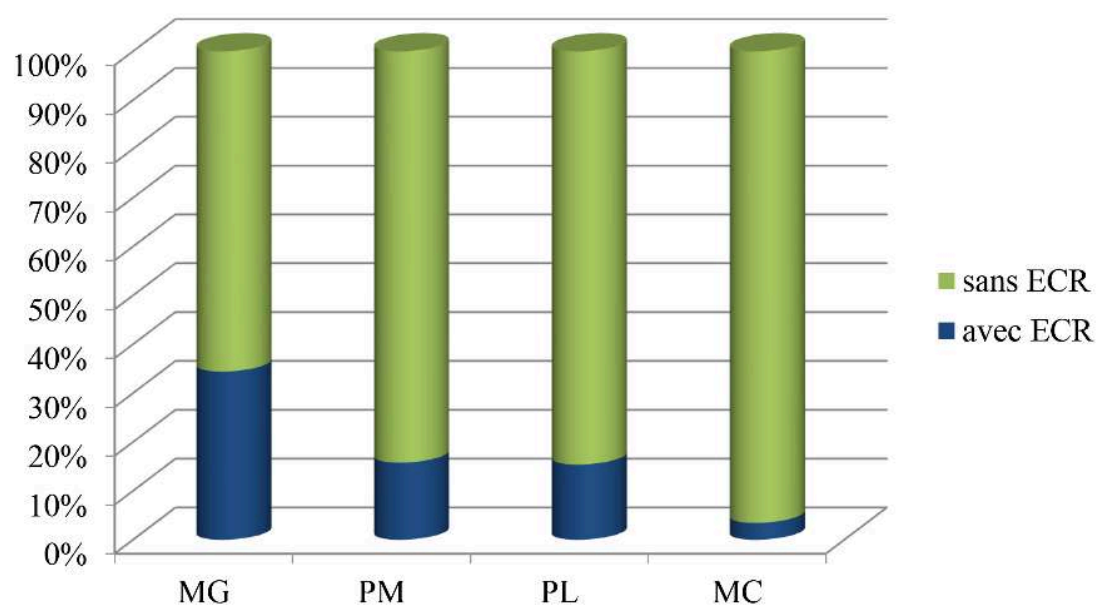




\section{Céramique faite à la main}

6 La céramique faite à la main, qui rassemble la majorité des pots de la maison MI, présente une grande variabilité quant aux pratiques techniques impliquées. Bien que son analyse demande encore à être affinée, on peut d'ores et déjà formuler quelques remarques préliminaires. Tout d'abord, deux techniques de façonnage différentes ont été identifiées. La majorité des pots semble relever de la technique du façonnage aux colombins, qui présente toutefois une grande variabilité au regard de son application ; on a ainsi observé différents modes de jointure des colombins (horizontale, en biseau, etc.) (fig. 5). Mais le matériel a aussi livré quelques exemples de la technique du façonnage par plaques, qui se caractérise par l'utilisation de masses d'argile préfabriquées en forme de plaques; cette technique est utilisée surtout pour les pots de grande taille, comme les jarres (fig. 6). D’autres pratiques ont aussi été observées au sein de la céramique faite à la main. L'une, très fréquente, tant parmi les pots fins que les pots grossiers, est l'usage d'un mouvement rotatif pour la régularisation des surfaces, ce qui suggère l'utilisation d'un support au cours du façonnage (fig. 7). Une autre pratique populaire concerne le façonnage des fonds: une part importante des pots, qu'ils soient de grande ou de petite taille, présente des fonds faits aux colombins renforcés par une plaque d'argile, ce qui explique la fréquence élevée des bases épaisses (fig. 8a-b). La céramique faite à la main comprend tous les types morpho-stylistiques présents au sein de la céramique élaborée au tour, ce qui suggère leur reproduction parallèle par différentes chaînes opératoires fondées sur des savoir-faire distincts. Plus précisément, la majorité des pots appartenant aux catégories des céramiques à peinture mate et à peinture lustrée est façonnée à l'aide de techniques manuelles, l'ECR n'étant utilisée que pour le façonnage d'une partie très limitée de la production. En revanche, la céramique minyenne semble se partager entre techniques manuelles (en particulier celle du façonnage aux colombins) et techniques exploitant l'ECR. La seule catégorie céramique qui, d'après les données actuelles de l'étude, ne fournit pas d'indice d'usage de l'ECR est la catégorie noire lissée. Cette catégorie implique l'établissement de chaines opératoires plus ou moins cohérentes fondées sur des choix techniques précis: usage exclusif des techniques manuelles sans l'intervention de l'ECR, avec prédominance du façonnage aux colombins, engobage en noir suivi par un polissage des surfaces, cuisson oxydante conférant au biscuit des couleurs rouges. 
Fig. 5. Tesson grossier 83/535.42.

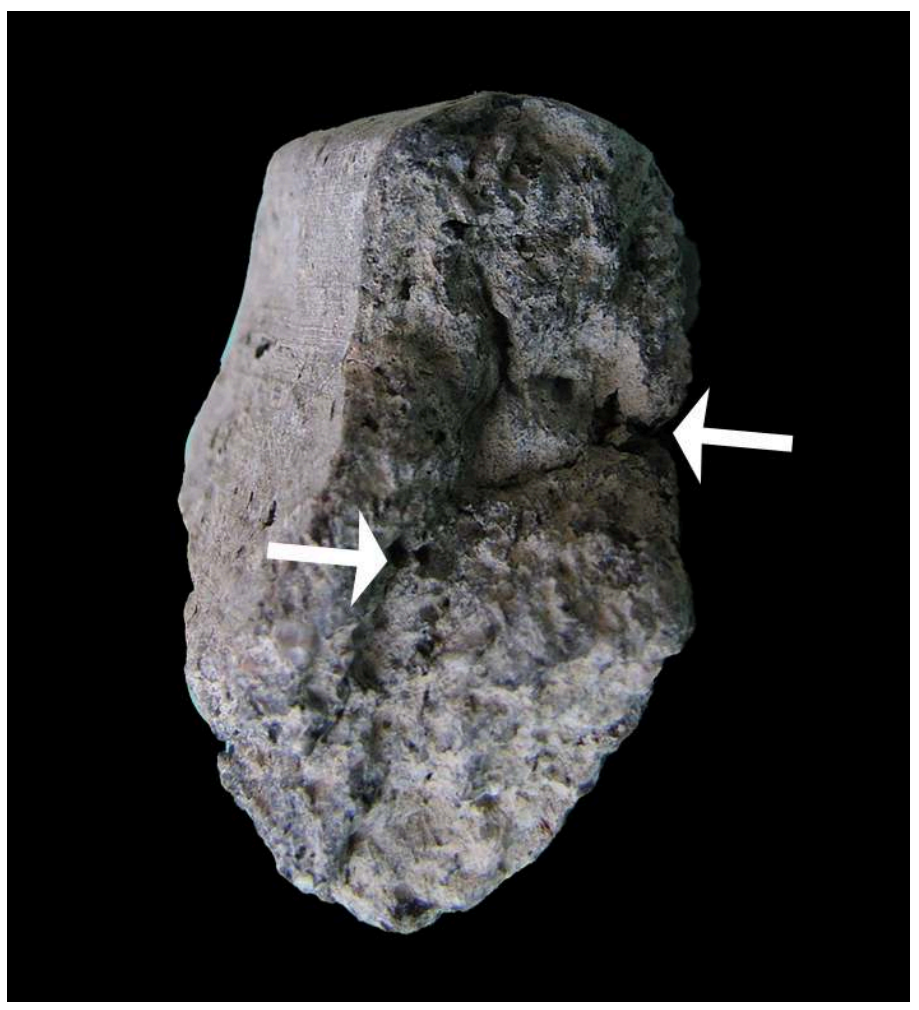

Section, jointure en biseau de deux colombins.

EFA.

Fig. 6. Tesson à peinture mate 11/319.40.

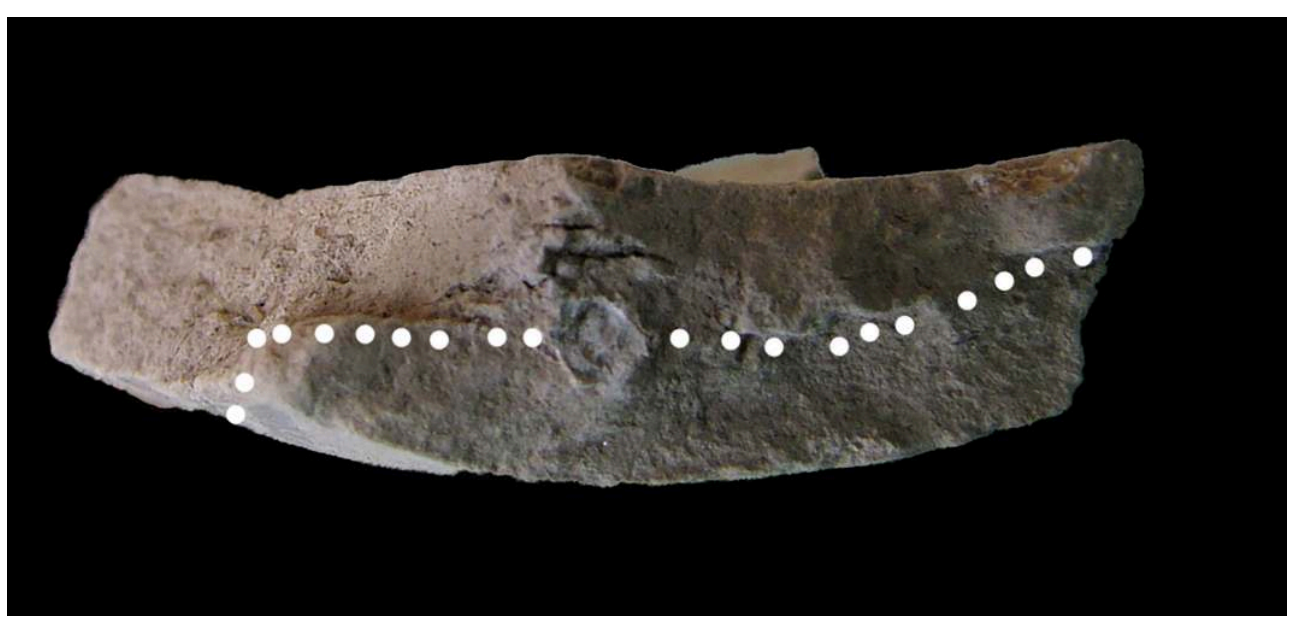

Section, jointure de deux plaques d'argile.

EFA. 
Fig. 7. Tesson grossier 11/318.8.

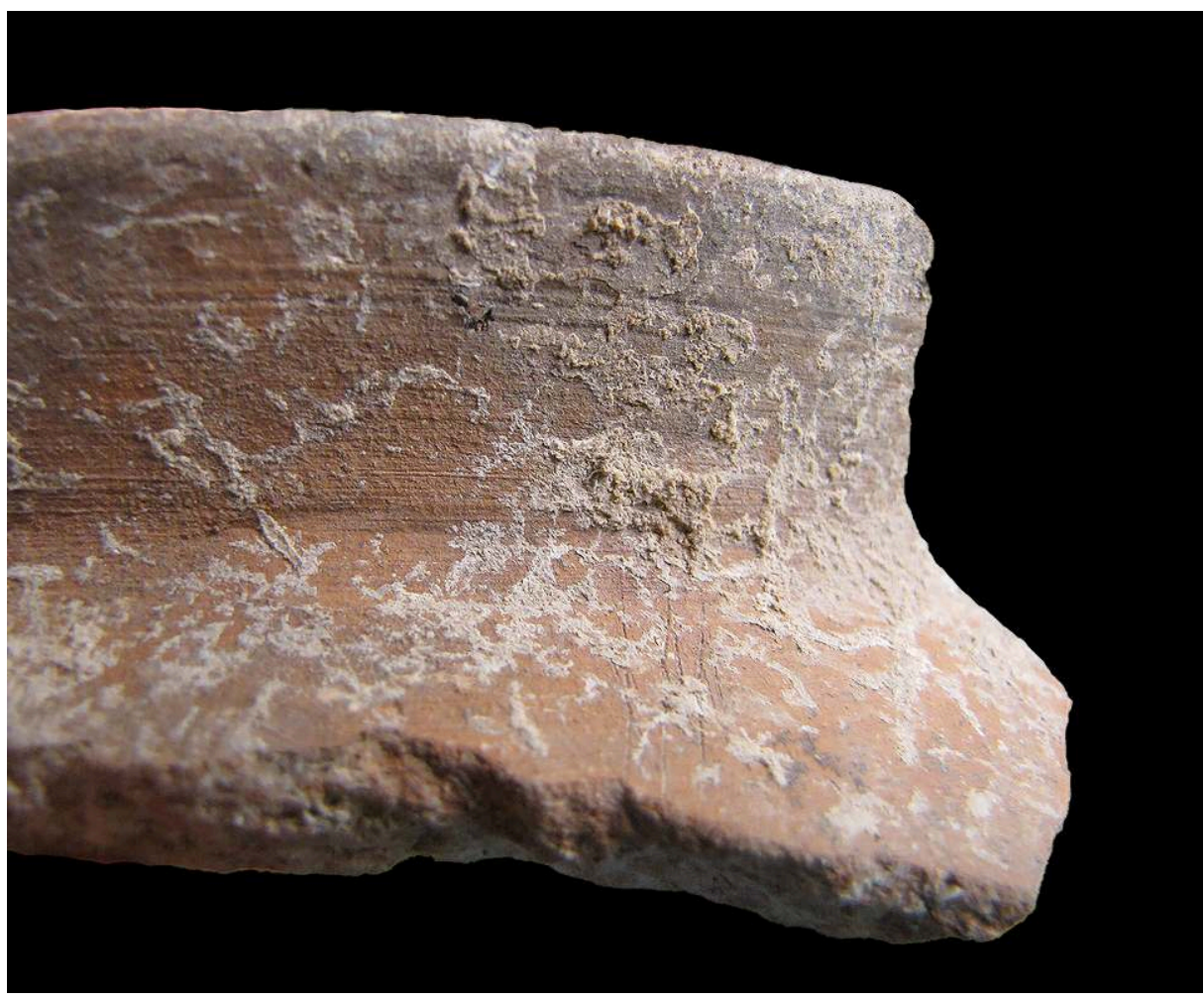

Surface externe, stries horizontales non rectilignes issues d'un mouvement rotatif. EFA. 
Fig. 8. a) Tesson minyen $83 / 531.19$; b) Tesson grossier 79/396.6.
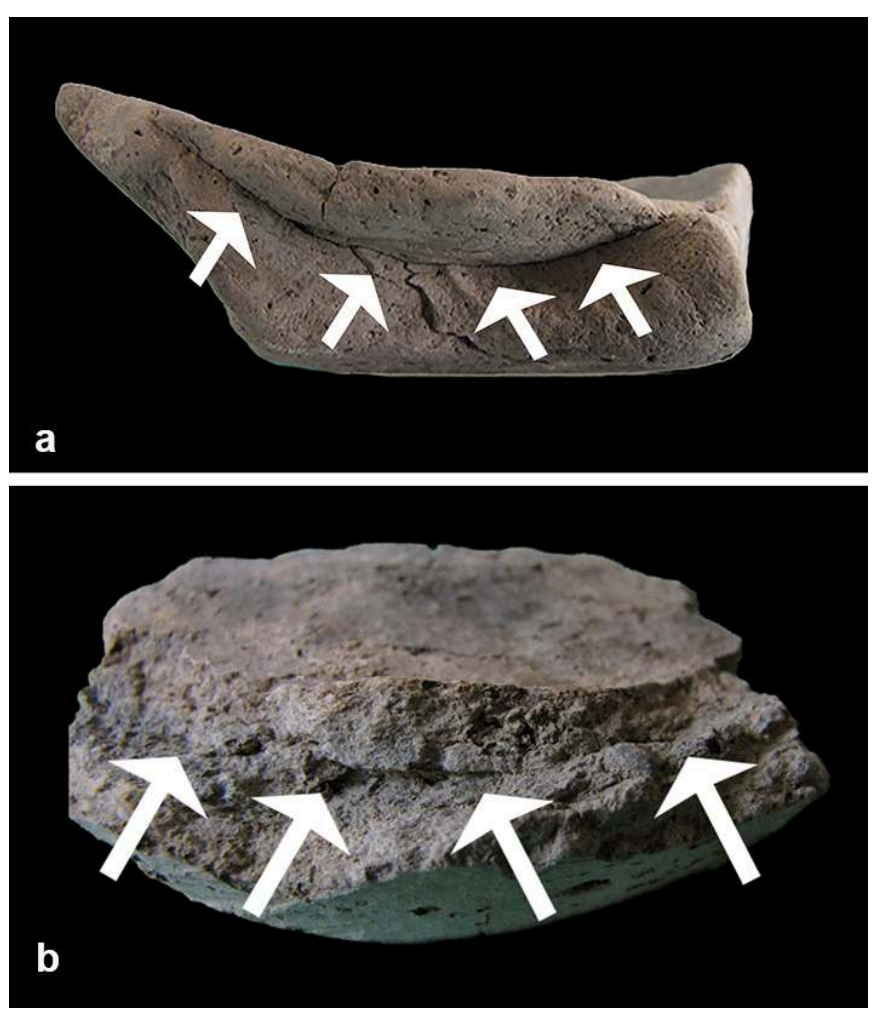

Sections, jointure horizontale d'éléments assemblés. EFA.

\section{La céramique de la « maison fantôme »}

7 L'étude de cet assemblage céramique - qui, contrairement au précédent, comprend des pots bien conservés - a porté exclusivement sur la céramique présentant de traces de l'ECR. Celle-ci ne semble pas dépasser $20 \%$ de l'assemblage ${ }^{9}$. Comme dans le cas du matériel de la maison MI, l'ECR est exploitée uniquement pour la mise en forme d'ébauches préalablement montées aux colombins (fig. 9-10) et son usage se limite parfois à l'élaboration des parties supérieures. Un seul pot fournit des traces indiquant l'emploi de l'ECR à partir d'une opération primaire du façonnage, à savoir celle de la jonction des colombins (fig. 11). Les chaînes opératoires qui sont liées à l'usage du tour se caractérisent par une uniformité technologique forte, la majorité des pots partageant des pratiques techniques similaires: usage de l'ECR pour l'ébauchage, traitement des surfaces par lissage à l'aide de l'ECR suivi par un polissage sans ECR, usage de pâtes fines et bien épurées, cuisson réductrice conférant aux surfaces et au biscuit une couleur grise uniforme (quelques surfaces ont une couleur grise ou brune non uniforme). La typologie morphologique consiste en formes ouvertes de petites dimensions : canthare galbé ou caréné, jatte carénée à épaule cannelée et bol renflé ou pansu, le dernier étant le plus populaire. Tous les pots élaborés au tour appartiennent à la céramique minyenne grise (fig. 12). 
Fig. 9. Tesson minyen 77/304.6.

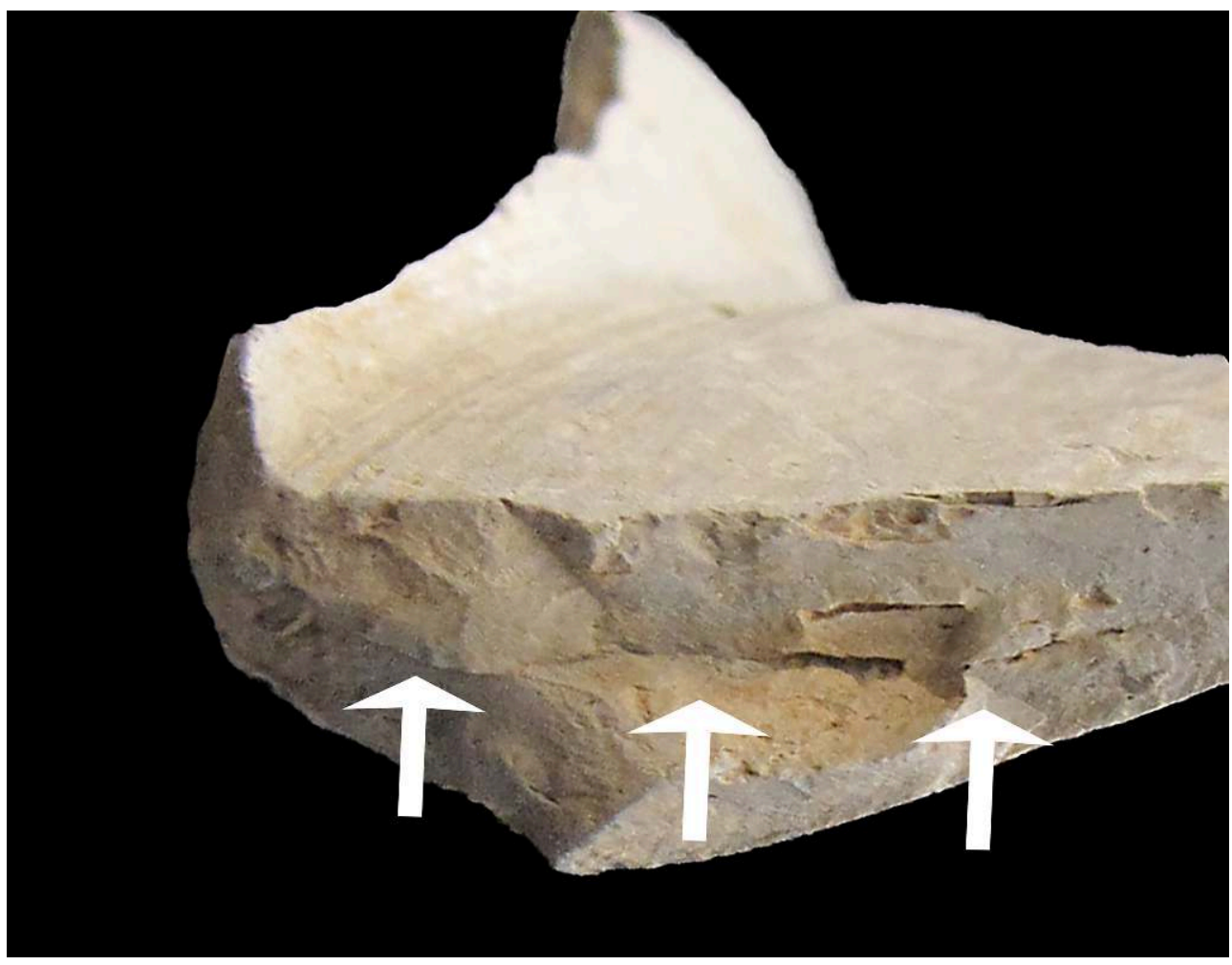

Section, joint de colombins.

EFA. 
Fig. 10. Tesson minyen 90/511.12.

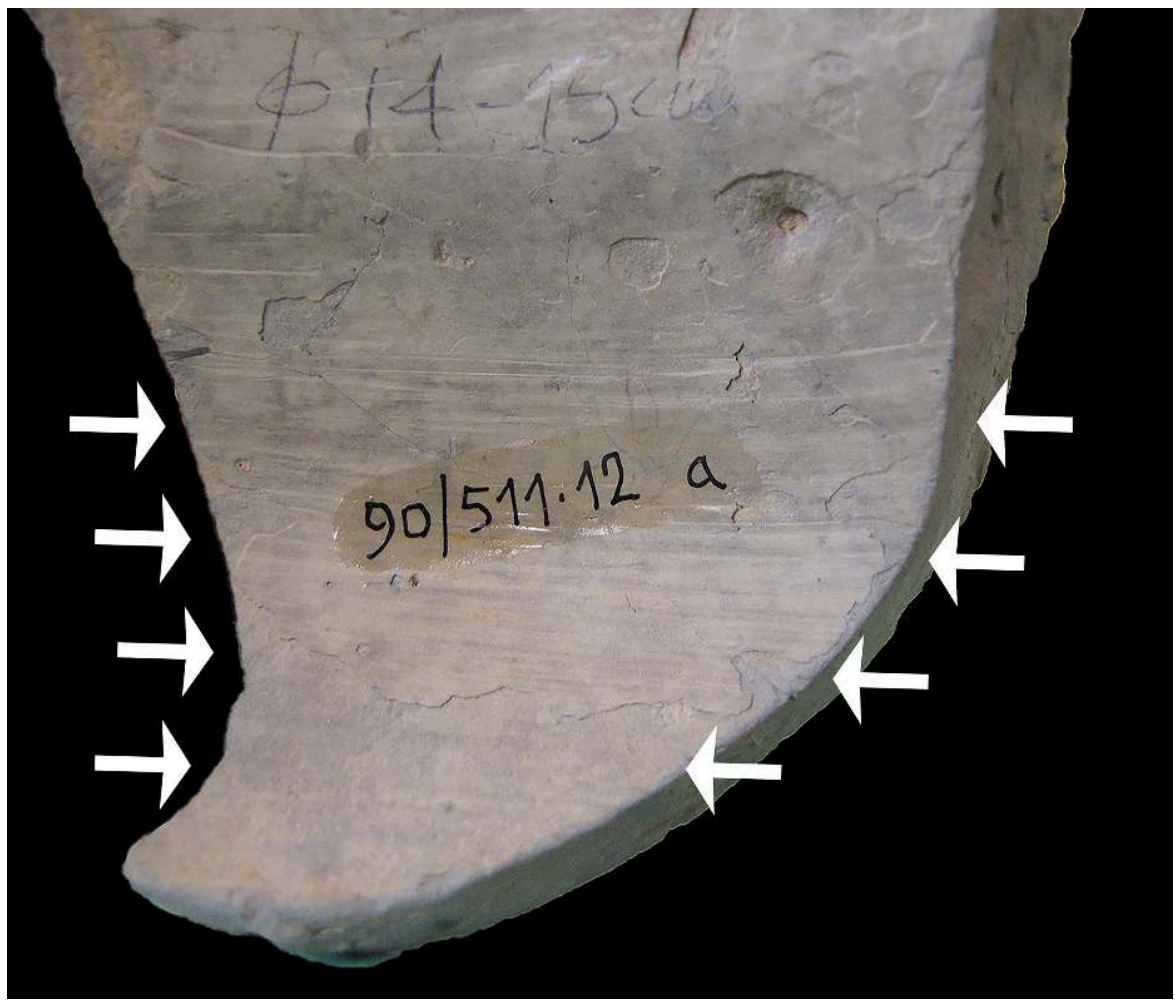

Surface interne, stries horizontales parallèles issues de l'ECR et microrelief irrégulier issu des pressions discontinues.

EFA.

Fig. 11. Tesson minyen 90/511.2.

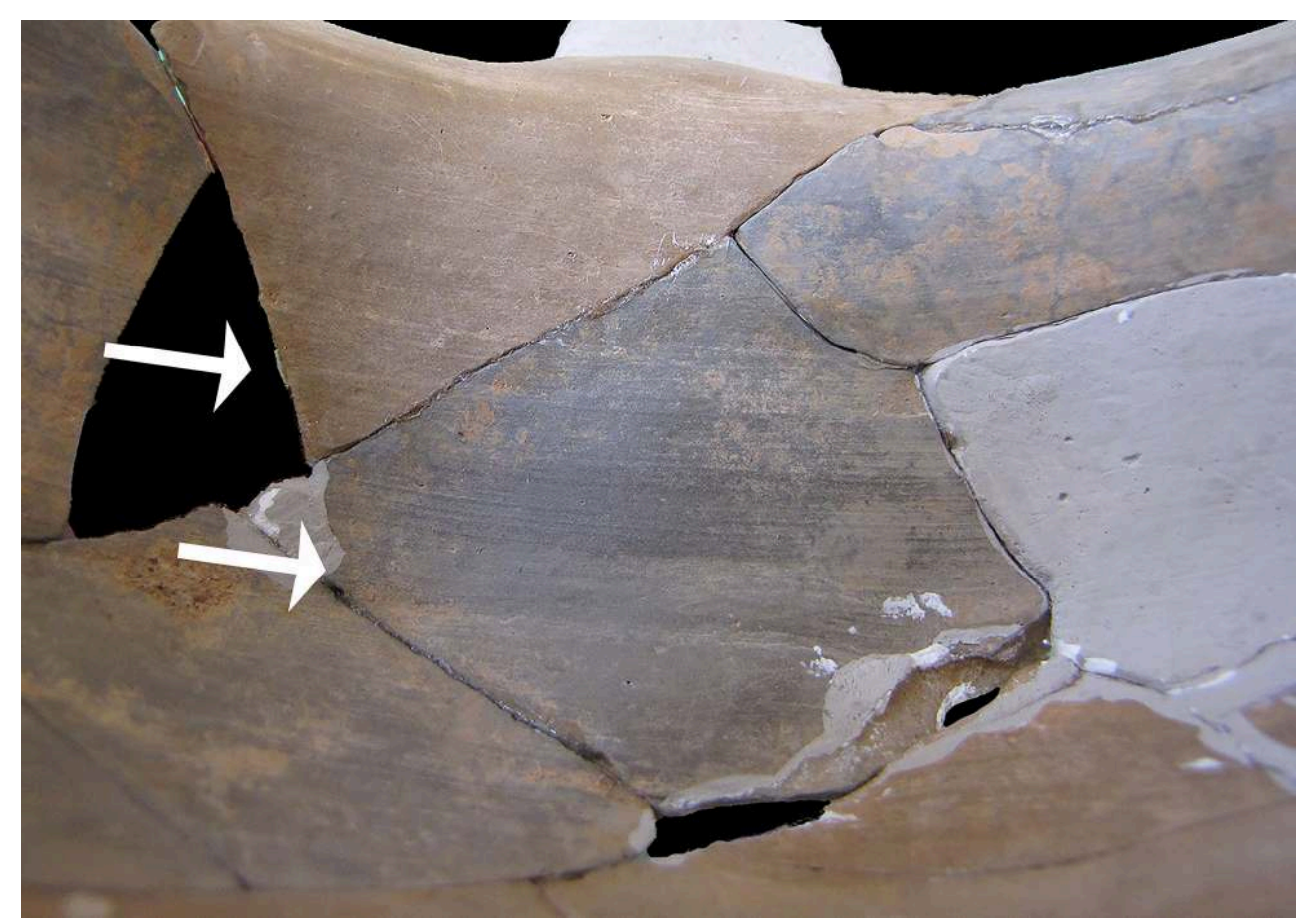

Surface interne, ondulations concentriques issues de l'ECR. EFA. 
Fig. 12. Types morphologiques de la céramique élaborée au tour de la « maison fantôme ».

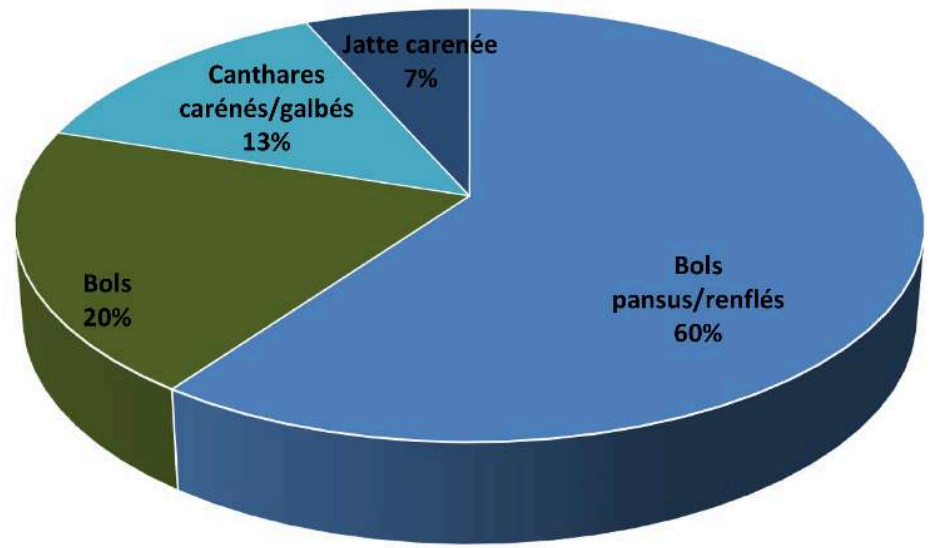

EFA.

Pour récapituler, il semble que le tour de potier fait sa première apparition sur l'Aspis pendant l'HMI, au sein d'une variété des petits bols à deux anses en céramique minyenne grise. Au cours de l'HMII, son usage concerne toujours les bols minyens, le canthare caréné devenant prédominant. En même temps, il bascule de façon sporadique à certaines autres formes, comme la jatte en céramique à peinture mate et la jarre à col resserré en céramique à peinture lustrée, où il a un usage sélectif qui se limite à l'élaboration finale des parties supérieures des pots. Toutefois, le tour de potier demeure un choix technique minoritaire tout au long des phases anciennes de l'habitat, sans marginaliser les techniques manuelles qui relèvent apparemment de traditions technologiques fortes et bien établies. Cependant, il est utilisé de façon constante, selon une méthode de façonnage spécifique, ce qui pourrait suggérer la transmission du même savoir-faire dans le temps. Un tel processus peut être identifié au sein de la céramique minyenne grise, qui partage plus ou moins les mêmes caractères technologiques systématiquement liés à la maîtrise du tour de potier.

Bien qu'une comparaison entre les deux assemblages étudiés se heurte au problème de leur état de conservation respectif très différent, elle permet néanmoins d'entrevoir certaines différences quant au choix technique lié au tour de potier et, par là même, aux unités de production sous-jacentes. La céramique élaborée au tour du petit assemblage de la "maison fantôme » est, dans sa majorité, le produit d'un savoir-faire standardisé ; elle représente un pourcentage assez élevé dans l'assemblage, suggérant l'activité d'une unité de production distincte qui se caractérise par une connaissance technologique particulière et cohérente en termes de choix impliqués dans la fabrication. En revanche, la céramique élaborée au tour de la maison MI, quoique peu 
abondante, se caractérise par une grande variabilité aussi bien du point de vue du mode d'emploi du tour de potier que de la diversité des catégories céramiques qui lui sont liées. Cet usage du tour de potier d'un caractère asystématique, combiné avec une variabilité au sein de la céramique faite à la main, suggère une différenciation au niveau des unités de production sous-jacentes, qui semblent fondées sur des savoirfaire et des choix technologiques variés. L'achèvement de l'étude du matériel de la " maison fantôme » et d'autres assemblages céramiques des secteurs Nord et Sud-Est, ainsi qu'une meilleure compréhension des traditions fondées sur les techniques manuelles, devraient permettre d'élucider l'impact du tour de potier sur le système technologique local ainsi que les voies par lesquelles il s'y est enraciné. À plus long terme, cela devrait apporter un éclairage nouveau sur la spécialisation artisanale et son rôle dans l'organisation sociale de la production au cours de l'Helladique Moyen.

\section{BIBLIOGRAPHIE}

COURTY, ROUX 1995

Marie-Agnes Courty, Valentine Roux, « Identification of wheel throwing on the basis of ceramic surface features and microfabrics », JAS 22, 1995, p. 17-50.

ROUX, COURTY 1998

Valentine Roux, Marie-Agnes Courty, « Identification of wheel-fashioning: technological analysis of 4th-3rd millennium B.C. Oriental ceramics », JAS 25, 1998, p. 747-763.

\section{NOTES}

1. Voir BCH 139-140, 2015-2016, p. 698.

2. Ce travail a été entrepris par J. Cutler (université de Cambridge), qui est malheureusement tombée gravement malade quelques mois plus tard et finalement décédée en janvier 2018 ; il a ensuite été repris par G. Ackermann et C. Martini.

3. Voir BCH 135, 2011, p. 554 et n. 4 ; Maria CHOLEVA, «L'apparition et la diffusion du tour de potier dans le monde égéen ", Thèse de doctorat, Université Paris 1 Panthéon-Sorbonne, 2015.

4. Voir BCH 138, 2014, p. 738-739, et fig. 10.

5. Nom provisoirement donné à la structure - non découverte - d'où provient le riche assemblage céramique trouvé sous la tombe sous-jacente à la maison absidale (BCH 102 [1978], p. 798); tout indique que cette structure fut détruite par le feu et ensuite nivelée.

6. Le matériel est très fragmenté. Ont été exclus de l'étude les tessons trop petits ou abîmés, ainsi que les fragments d'anses et les tessons qui ne fournissent pas d'informations sur les opérations liées au façonnage. Les tessons exclus représentent environ $30 \%$ de la totalité de la céramique étudiée. 
7. Sur cette technique hybride, COURTY, ROUX 1995 et ROUX, COURTY 1998, p. 747-763.

8. Les quantifications se fondent sur le matériel conservé.

9. Un ensemble de 75 tessons/pots a été étudié. Contrairement à la céramique de la maison MI, l'assemblage de la "maison fantôme " comprend de grandes pièces, souvent recollées.

\section{INDEX}

Thèmes : EFA

chronologie https://ark.frantiq.fr/ark:/26678/pcrtxT02uJOogm, https://ark.frantiq.fr/ark:/ 26678/pcrtGTWPtWn8qu

lieux https://ark.frantiq.fr/ark:/26678/pcrtRm0r24dwUy sujets https://ark.frantiq.fr/ark:/26678/pcrtbptj4SOA1W, https://ark.frantiq.fr/ark:/26678/ pcrttNvr7jGBQI, https://ark.frantiq.fr/ark:/26678/pcrt1DMOWvDF4j

Année de l'opération : 2016

\section{AUTEURS}

\section{ANNA PHILIPPA-TOUCHAIS}

UMR 7041 « Archéologies et Sciences de l'Antiquité (ArScAn)»

\section{GILLES TOUCHAIS}

Université Paris 1, UMR 7041 « Archéologies et Sciences de l'Antiquité (ArScAn)»

\section{MARIA CHOLEVA}

Université de Louvain-la-Neuve 